\author{
Lawrence R. Kuhns \\ Randall T. Loder \\ Evelyn Rogers \\ Donna Anderson
}

\section{Head-wag autotomography of the upper cervical spine in infantile torticollis}

low-radiation-dose procedure. Conclusion. For infants with torticollis in whom vertebral anomalies need to be excluded, the "head wag" technique is useful.
L. R. Kuhns $(-$ E. Rogers $\cdot$ D. Anderson Department of Pediatric Radiology, University of Michigan Hospitals, 1500 E. Medical Center Drive, Ann Arbor, MI 48109, USA

R. T.Loder

Department of Orthopaedic Surgery, University of Michigan Hospitals, Ann Arbor, Michigan, USA

\begin{abstract}
Background. Infants with torticollis require evaluation of the entire cervical spine for vertebral anomalies as part of the work-up, but open-mouth views to assess occiput to $\mathrm{C} 2$ are difficult to obtain. Objective. To evaluate a "head wag" autotomographic technique for the frontal projection of this region. Materials and methods. Twenty infants under one year of age were evaluated using this technique. Results. Sixteen of 20 could be adequately evaluated using this simple,
\end{abstract}

\section{Materials and methods}

From February 1996 to October 1997, 20 infants with torticollis were evaluated using the "head wag" method of autotomography. During 1996 this method was used when a standard odontoid view failed. By 1997 we stopped performing the routine view in these patients and the head wag technique was the only method employed. To perform this technique, the infant's body is immobilized using book ends and sand bags [4], and the head is placed on a circular sponge with a hole in the middle. The occiput rests on the sponge just above the tabletop. The examiner wears a lead apron and lead gloves, and the gloved hands are placed on each side of the head. The head is gently rocked about $1 \mathrm{~cm}$ side-to-side during an exposure in which the milliamperage has been reduced and the length of exposure has been increased so that the skin entrance dose remains the same as for a routine odontoid view. Typical factors for Bucky technique using our nominal 400-speed screen-film combination (Kodak Lanex regular film with Lanex medium speed screens) are $50 \mathrm{kVp}, 10 \mathrm{~mA}$, and $3.6 \mathrm{~s}$ exposure time. The focal film distance is 40 in. $(102 \mathrm{~cm})$ and the small focal spot is used. The head is rotated 3 to 4 times during the exposure. The infant must be relaxed at the time of exposure, which may require a pacifier or bottle-feeding just before the exam is initiated. There is no angulation of the tube or the tabletop if the neural arches or the occipito-atlantal joints need to be visualized (Fig. 1). If the vertebral bodies need to be evaluated, a 10 to $15^{\circ}$ cephalic angulation of the tube is helpful [5]. 

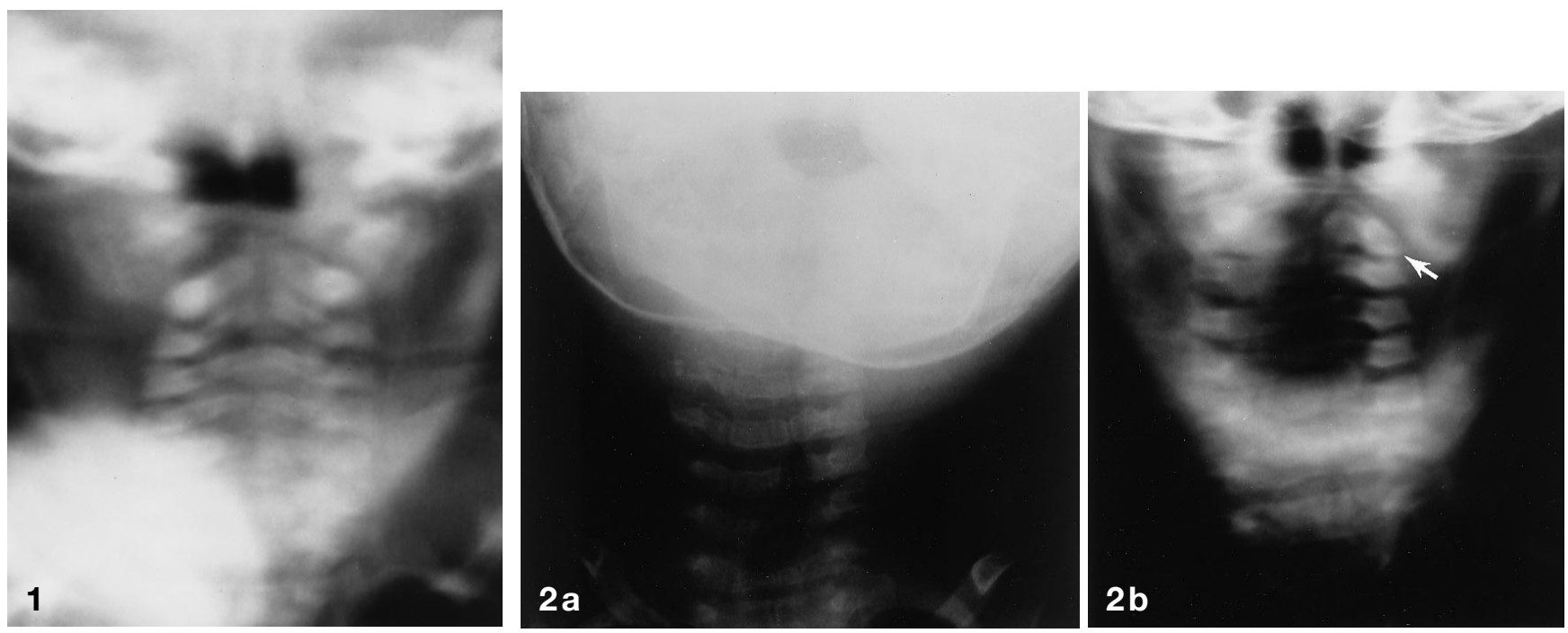

Fig.1 Head wag radiograph of a normal infantile cervical spine. The $\mathrm{C} 1$ lateral masses are the most blurred, since most of the rotation is occurring at $\mathrm{C} 1$. The radiographic tube was not angled in relation to the tabletop, which allows optimal visualization of the neural arches in the infant. The pacifier is seen overlying the lower right cervical spine

Fig. 2 a On this frontal radiograph, the occiput and the chin are tilted to the left. b On this autotomogram, there is hypoplasia of the articular pillar of $\mathrm{C} 2$ on the left (white arrow)

\section{Results}

Four of the exams were technically inadequate early in our experience because the infant was crying and moving the head in other directions besides rotation. Since we initiated use of a pacifier or bottle-feeding at the time of the exposure, all exams have been successful (Fig.1). One patient with Klippel-Feil syndrome had multiple vertebral anomalies of the entire cervical spine. The neural arch defects of $\mathrm{C} 1-\mathrm{C} 3$ were quite well seen on the autotomogram. One infant presented soon after birth with the head tilted to the left (Fig.2). On the autotomogram, hypoplasia of the left articular pillar of $\mathrm{C} 2$ was seen (Fig.2), and this correlated well with the position of the head. This neural arch hypoplasia could not be visualized on the lateral radiograph. This patient may have had familial cervical dysplasia [1].

Another infant presented with the occiput tilted to the left (Fig. $3 \mathrm{a}$ ) and the chin persistently extended (Fig. 3 b). On the autotomogram the left lateral mass of $\mathrm{C} 1$ appeared to project above the foramen magnum (Fig. 3c). One-millimeter-thick axial CT scans were performed through the cranio-cervical junction and coronal reformatting was performed. On a posterior reformatted image, the left neural arch of $\mathrm{C} 1$ appeared to project
Table 1 Differential diagnosis of torticollis

\section{Congenital}

Occipitocervical anomalies

Basilar impression - primary

Atlanto-occipital anomalies

Asymmetry of occipital condyles

Unilateral absence of $\mathrm{C} 1$ facet

Odontoid anomalies (aplasia, hypoplasia, os odontoideum)

Klippel-Feil syndrome

Familial cervical dysplasia

Pterygium colli

Congenital muscular torticollis

Acquired

Basilar impression - secondary

Idiopathic/inflammatory

Atlantoaxial rotary displacement, subluxation, fixation

Neurogenic

Spinal cord tumors

Cerebellar tumors, posterior fossa tumors

Syringomyelia

Ocular dysfunction

Bulbar palsies

Arnold-Chiari malformation

Inflammatory

Cervical adenitis, Grisel's syndrome, retropharyngeal abscess

Juvenile rheumatoid arthritis, rheumatoid arthritis

Disc space calcification

Tuberculosis

Neoplasm

Osteoid osteoma

Aneurysmal bone cyst

Sandifer's syndrome

(Reprinted by permission from [1])

into the foramen magnum (Fig. $3 \mathrm{~d}$ ). On a reformatted image of the mid-foramen magnum the ossified left lateral mass of $\mathrm{C} 1$ was somewhat underdeveloped as compared to the right and was tilted to the left (Fig. 3e). On a coronal image through the odontoid, the ossified 

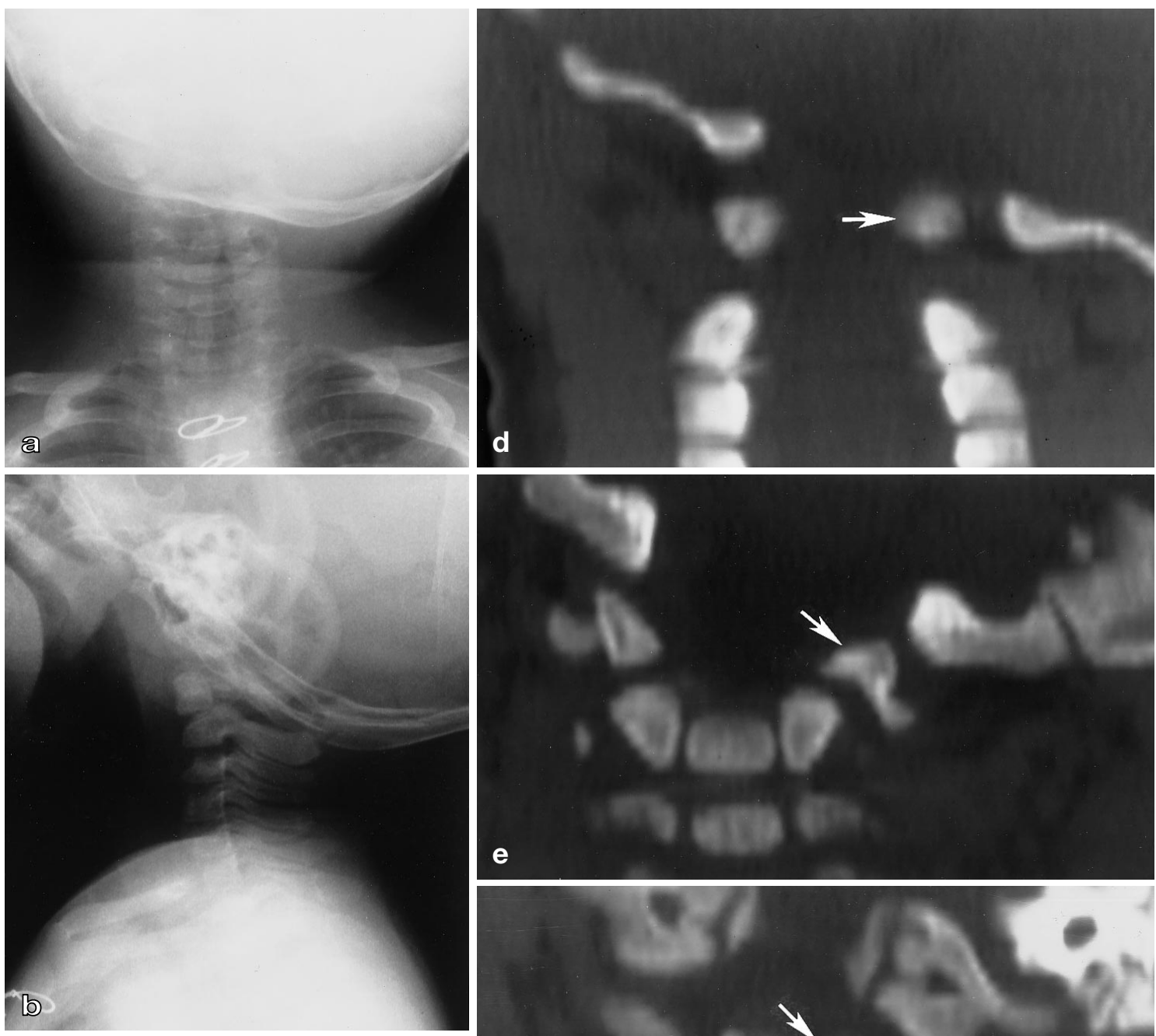

e
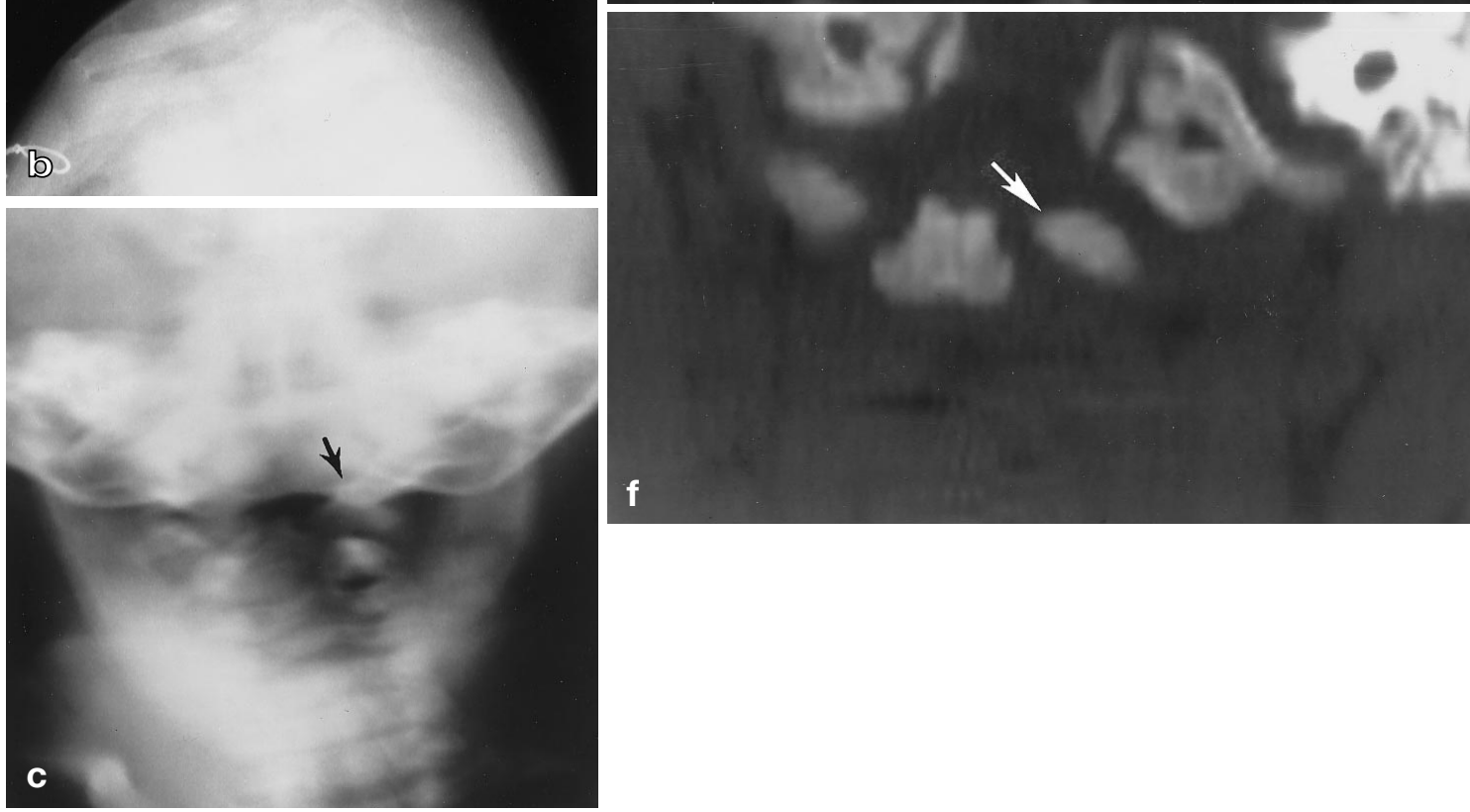

Fig.3 a The occiput is tilted to the left. b The occiput remained extended on $\mathrm{C} 1$, even when attempts were made to flex the cervical spine. c On the autotomogram, the left lateral mass of $\mathrm{C} 1$ appears to project above the foramen magnum (black arrow). d On this coronal reformatted image from the CT data set through the posterior foramen magnum, the left posterior neural arch of $\mathrm{C} 1$

projects to a level parallel to the left lip of the foramen magnum (white arrow). e At the level of the mid-foramen magnum the ossified lateral mass of $\mathrm{C} 1$ appears hypoplastic compared to the right lateral mass. The left mass is tilted laterally (white arrow). f On this coronal image at the level of the odontoid the ossified left lateral mass of $\mathrm{C} 1$ is still hypoplastic and tilted (white arrow) 
left lateral mass of C1 still appeared hypoplastic and tilted (Fig. $3 \mathrm{f}$ ). This patient probably had hypoplasia of the left occiput with left unilateral basilar invagination [6].

\section{Discussion}

The referring physician usually palpates the sternocleidomastoid muscles carefully for tightness, shortening, or a mass-like deformity [7]. If the sternocleidomastoid exam is unremarkable, the patient may be referred for skull and spine radiographs. Synostosis of the lambdoid suture(s) is evaluated by careful analysis of the skull radiographs. Negative spine radiographs are important in eliminating the occipito-cervical spine as a cause of torticollis. Evaluation of the occiput-C2 complex on the frontal projection using a radiographic technique rather than linear tomography or CT is less expensive and requires less radiation exposure to the patient. Our preliminary experience would suggest that the technique of Li and Paull seems well suited for elimination of occipito-cervical vertebral anomalies as a cause of infantile torticollis. When abnormalities are seen using this technique, CT with reformatting may be used to confirm and further define the anomaly. When cervical radiographs are negative, attention can be directed to other possible causes of torticollis, such as brain tumors or Sandifer's syndrome (Table 1).

\section{References}

1. Loder RT, Hensinger RN (1994) Developmental abnormalities of the cervical spine. In: Weinstein SL (ed) The pediatric spine: principles and practice. Raven Press, New York, pp 397-420

2. Swischuck LE (1996) Pursuing the odontoid fracture in infants (guest editorial). Emerg Radiol 3: 54-55
3. Li CP, Paull D (1971) A new head rotation method for cervical spine radiography. Radiology 98: 568

4. Poznanski AK (1976) Practical approaches to pediatric radiology. Year Book Medical Publishers, Chicago, pp 21-30

5. Abel MS (1983) Occult traumatic lesions of the cervical and thoraco-lumbar vertebrae (with an evaluation of the role of CT). 2 nd ed. Warren H. Green, St. Louis, MO, pp 18-20
6. Wackenheim A (1974) Roentgen diagnosis of the craniovertebral region. Springer, Berlin Heidelberg New York, pp 355-359

7. Cheng JCY, Au AWY (1994) Infantile torticollis: a review of 624 cases. J Pediatr Orthop 14: 802-808 